\title{
Treatment of Irreducible Bilateral Cervical Facet Fracture-Dislocation with a Prolapsed Disc Using a Prefixed Polyetheretherketone Cage and Plate System
}

\author{
Kyung-Jin Song ${ }^{1}$, Hun Park ${ }^{2}$, Kwang-Bok Lee ${ }^{1}$ \\ ${ }^{1}$ Department of Orthopedic Surgery, Research Institute of Clinical Medicine of Chonbuk National University-Biomedical Research Institute of \\ Chonbuk National University Hospital, Chonbuk National University Medical School, Jeonju, Korea \\ ${ }^{2}$ Department of Orthopedic Surgery, Gwangju Veteran Hospital, Gwangju, Korea
}

When anterior reduction fail in the surgical treatment of cervical bilateral facet fracture-dislocation with concomitant disc extrusion, it is necessary to perform a reduction using a posterior approach and then a third anterior procedure is often necessary to accomplish the anterior reconstruction. This presents difficulties for both patients and surgeons because of the need for frequent position changes (supine-prone-supine). The purpose of this study is to illustrate a modified surgical technique, which is anterior reduction and fixation with a prefixed polyetheretherketone (PEEK) cage to a buttress plate for the treatment of irreducible bilateral cervical facet fracture-dislocation with a prolapsed disc is an enhancing technique for the stability of the interbody graft than a buttress plate alone because the PEEK cage has more fixation power and reduces both the number of position changes and the length of the operation.

Keywords: Fracture dislocation; Cage; Anterior reduction

\section{Introduction}

Distractive flexion injury is the most common injury of the lower cervical spine [1]. These injuries present as the subluxation or the unilateral or bilateral dislocation of the facet joints, which may be accompanied by direct spinal cord and nerve root injury [2]. Decision making regarding the approach to treatment of a facet joint fracture or dislocation requires careful evaluation of variables such as the need for reduction and the appropriate timing, the presence of herniated intervertebral discs, and the degree of damage to the posterior ligament complex. When an anterior reduction fails after discectomy, it should be attempted again using a posterior approach, and then the fixation should be performed through an anterior approach. This method (supine-prone-supine) creates difficulties for surgeons because of the prolonged operation time; the need for frequent changes in patient position; and a subsequent high incidence of complications, such as infection and hemodynamic instability.

An anterior buttress plate technique [3] can eliminate the need for additional anterior fixation after a reduction through a posterior approach. However, the buttress plate does not provide safety from graft motion or impinge-

Received Feb 22, 2012; Revised Apr 25, 2012; Accepted May 9, 2012

Corresponding author: Kwang-Bok Lee

Department of Orthopedic Surgery, Chonbuk National University Hospital, Chonbuk National University Medical School, 776 1sunhwan-ro, Heungdeok-gu, Cheongju 361-711, Korea

Tel: +82-63-250-1760, Fax: +82-63-271-6538, E-mail: osdr2815@naver.com 
ment of the spinal cord since it does not completely fix the interbody graft. Therefore, we present our experience with a modified technique using a prefixed cage to the buttress plate without the additional anterior approach.

\section{Technical Note}

A 26-year-old man presented with bilateral motor weakness of the upper and lower extremities due to an automobile accident. On physical examination, motor strength for the upper extremities was as follows: deltoid, $2 / 2$; biceps, $2 / 1$; wrist extensor, $1 / 1$; triceps, $1 / 2$; finger flexor, $1 / 1$; finger abductors, $1 / 1$. Motor strength for the upper extremities was as follows; iliopsoas, $1 / 1$; quadriceps, $1 / 1$; tibialis anterior, $2 / 0$; extensor hallucis, $1 / 0$; ankle plantar flexor, $1 / 0$. Sensory examination

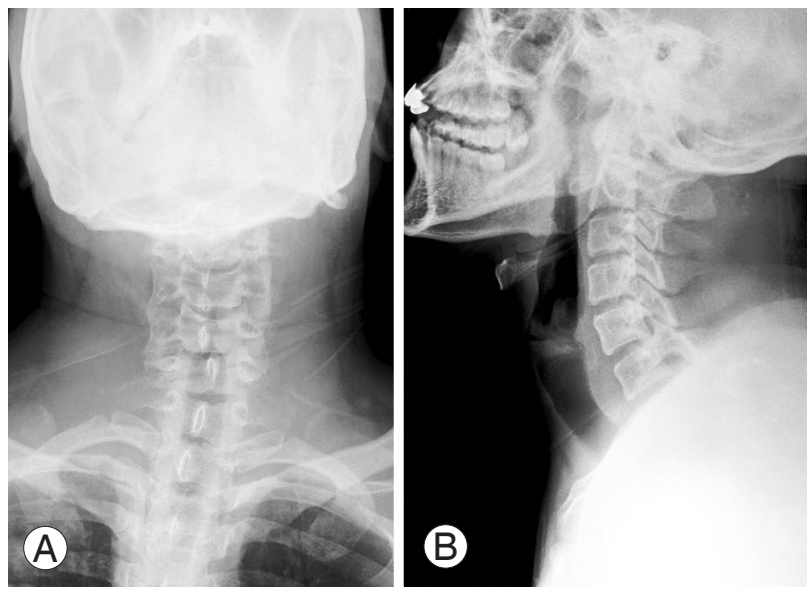

Fig. 1. Preoperative $\mathrm{C}$-spine anteroposterior $(\mathbf{A})$ and lateral $(\mathbf{B})$ plain radiographs showing mal-alignment of the spinous processes of the fourth to sixth cervical vertebra and anterior dislocation of the $\mathrm{C} 6$ body to the $\mathrm{C} 7$ body. demonstrated an partial loss of pain and temperature sensation below the T4 dermatome. Babinski's reflex and ankle clonus were also noted. Plain anteroposterior and lateral radiographs demonstrated a malalignment of the spine from $\mathrm{C} 5$ to $\mathrm{C} 7$ without a lordotic curve loss and no bony fracture-dislocation from $\mathrm{C} 1$ to C6 (Fig. 1). Sagittal computed tomography (CT) scans revealed bilateral dislocation and locking of the facet joint between $\mathrm{C} 6$ and C7 (Fig. 2). T2-weighted magnetic resonance (MR) images revealed cord compression due to intervertebral disc herniation between $\mathrm{C} 6$ and $\mathrm{C} 7$ as well as between $\mathrm{C} 4$ and $\mathrm{C} 5$, and posterior ligament complex rupture and posterior neck muscle injury (Fig. 3). We evaluated the patient needed emergency decompression.

The patient was placed in the supine position. Using a Smith-Robinson anterior cervical approach, the disc was totally removed. The posterior longitudinal ligament was disrupted, and the ligament was resected to ensure adequate decompression. The superior and inferior endplates were ordinally prepared to accept the graft mate-
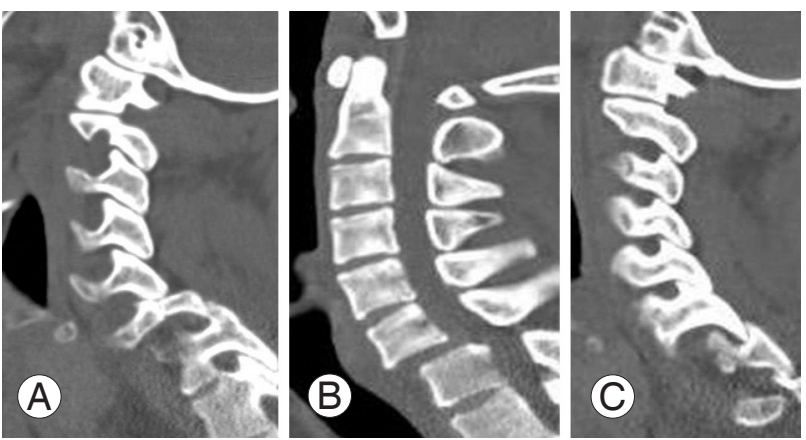

Fig. 2. Preoperative sagittal computed tomography showing dislocation and locking of the facet joints of the sixth and seventh cervical vertebra: (A) right facet cut, (B) midsagittal cut, and (C) left facet cut.

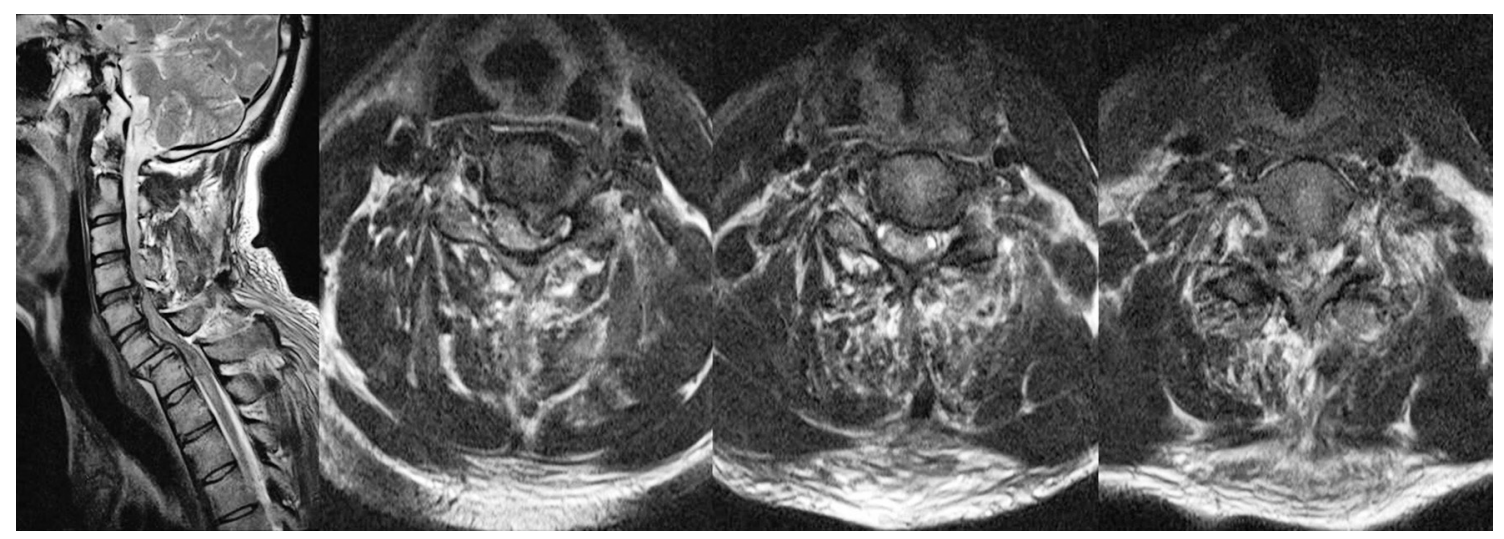

Fig. 3. Preoperative T2-weighted magnetic resonance images showing dislocation of the facet joints of the C6 and C7 vertebra and cord compression caused by prolapsed discs at C4-C5, C5-C6, and C6-C7. 
rial. We then attempted a reduction through an anterior approach but the reduction failed. We appropriately bent the cervical plate (Maxima, U\&I, Seoul, Korea) and prefixed a cage (Solis cage, Stryker Biotech, Hopkinton, MA, USA) to the plate using one screw (4 mm diameter, 12 $\mathrm{mm}$ length, Maxima, U\&I) which was placed into the C6C7 disc space to restore proper intervertebral height and lordosis (Figs. 4, 5). We then applied the prefixed cage and plate system from the bodies of $\mathrm{C} 4$ to $\mathrm{C} 7$ and fixed it using screws to the $\mathrm{C} 4, \mathrm{C} 5$, and $\mathrm{C} 6$ bodies but not to the $\mathrm{C} 7$ body. We noted that the $\mathrm{C} 6$ and $\mathrm{C} 7$ vertebral bodies remained in an unreduced state (Fig. 5A). The anterior wound was then closed, and the patient was carefully placed in the prone position for the posterior procedure. A midline posterior cervical incision was made and the posterior elements were exposed. A portion of the superior vertebral facet of $\mathrm{C} 7$ vertebra was resected to facilitate the reduction. Penfield (No. 4) were placed between the superior and inferior facets. Under fluoroscopic visualization, the dislocation was reduced by unlocking the facets and then extending the neck and translating the

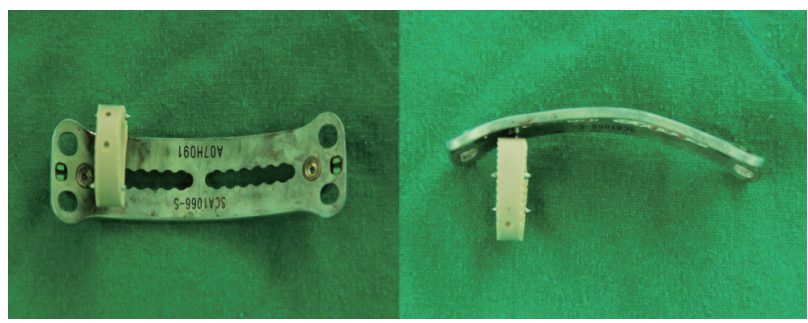

Fig. 4. Polyetheretherketone cage prefixed to the cervical plate using one screw through the central hole of the plate. superior dislocated segment posteriorly [3]. Fluoroscopy verified that there was no abnormal motion of the graft in the disc space at the location of the pre-fixation to the buttress plate, that the screws and plate remained secure, and that the graft did not impinge on the spinal cord. The posterior elements were then fused using lateral mass screws for C3-C5 and pedicle screws for C6-T1 to gain correct cervical lordosis. We confirmed that there was complete reduction at C6-C7 (Fig. 5B-5D). External immobilization was performed with a Philadelphia brace for six weeks.

At the follow-up assessment, the patient demonstrated no significant improvement in function or independence in activities of daily living. At the one-year follow-up, both the anterior and posterior cervical fusions were completely consolidated with the simple radiographs showing union, and there was no instability or hardwarerelated complications.

\section{Discussion}

Many articles have reported that a reduction in facet joint dislocations with accompanying intervertebral disc herniations should be performed after the herniated intervertebral discs have been removed through an anterior approach to prevent the exacerbation of neurologic symptoms $[2,3]$. If satisfactory results are achieved with a reduction after the removal of the herniated discs through an anterior approach, then an anterior fixation and fusion can be performed. An additional posterior fixation and fusion may also be performed depending on the patient's condition. In patients that are not successfully reduced
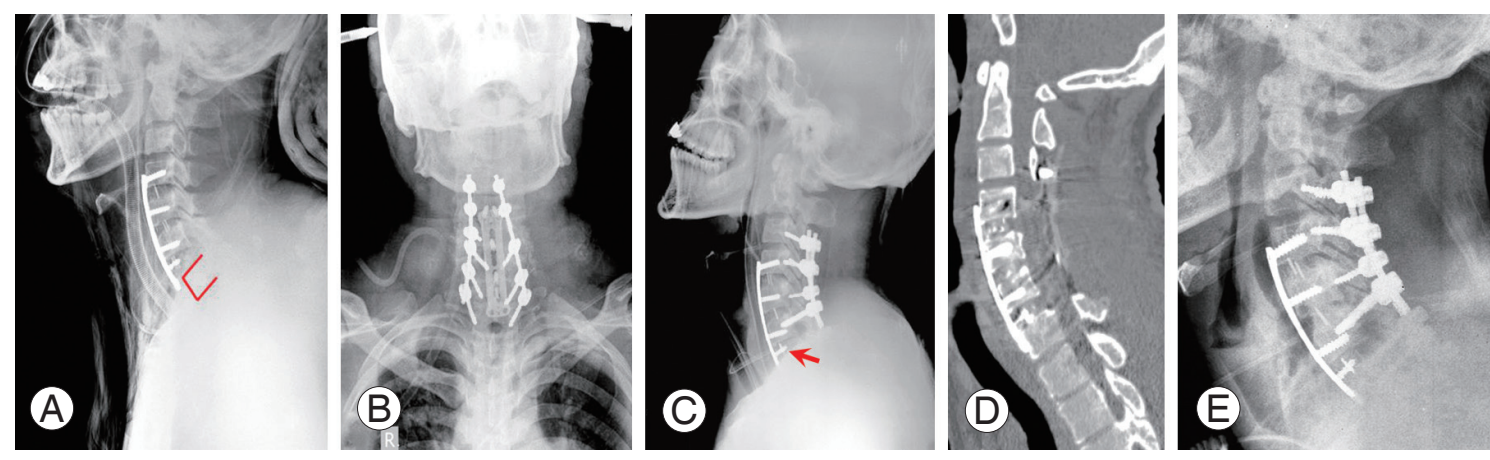

Fig. 5. Intraoperative $\mathrm{C}$-spine lateral (A) radiograph using the anterior approach showing a gap between the anterior aspect of the C6 body and C7 body because of the irreducible state. Immediate postoperative C-spine AP (B) and lateral (C) radiographs showing anterior plate fixation with polyetheretherketone (PEEK) cages at C4-C5 and C5-C6, a prefixed PEEK cage (red arrow) at C6-C7, and posterior fixation at C3-C7. The postoperative sagittal computed tomography scan (D) shows good alignment, complete reduction, and no malpositioning of the cage. The final follow-up C-spine lateral radiograph (E) showing bony fusion at C4-C7 and no hardware-related complications, such as graft malpositioning, metal breakage, or screw loosening. 
through an anterior approach, reduction is performed through a posterior approach followed by a fixation and fusion through an additional anterior approach [4-7]. In such cases, there is an increased risk of complications such as infection and hemodynamic instability because of the prolonged operation time and the frequent position changes. Allred and Sledge [3] used a technique that incorporated a buttress plate, which can obviate the need for additional anterior fixation after reduction through a posterior approach in cases when reduction has failed through an anterior approach. This method has the advantage of reducing the number of operations and the number of position changes because it avoids additional anterior fixation. Although the authors made a shelf to prevent graft dislodgment into the posterior space (spinal cord), a major disadvantage of this technique is that the buttress plate cannot completely be fixed to the interbody graft. Graft material can still remain dislodged anteriorly or posteriorly during a posterior reduction maneuver because reduction has failed through an anterior approach (Fig. 5A). To avoid this problem, a more reliable fixation method for the graft and plate system was needed.

We developed a prefixed cage to buttress plate that fixed one screw into the hole in the midspace of the polyetheretherketone cage to enhance the stability of the graft material. We then put the prefixed cage and plate system into the discectomy space of the dislocated cervical spine. It was fixed in place with screws to the cranial vertebral body (C6 vertebra but not the $\mathrm{C} 7$ vertebra) of the unreduced facet fracture for reduction through a posterior approach. Because the method that we have demonstrated stabilized the graft material, we believe that it can prevent unforeseeable complications that may arise due to instability of the graft material.

Although this method can improve the initial stability of the graft material, this cannot completely eliminate all of the instability because there is no fixation between the plate and lower vertebral body.

In cases of failed reduction through an anterior approach after discectomy for decompression in facet fracture-dislocation with accompanying intervertebral disc herniation, it may be possible to omit additional anterior fixation by using prefixed cage to the buttress plate. This technique might increase the stability of the interbody graft without compromising posterior reduction maneuver in an irreducible cervical fracture-dislocation with a prolapsed disc.

\section{Conflict of Interest}

No potential conflict of interest relevant to this article was reported.

\section{Acknowledgements}

This study was performed with the approval of the Institutional Review Board of the Chonbuk National University Research Council (IRB-2011-32).

\section{References}

1. Allen BL Jr, Ferguson RL, Lehmann TR, O’Brien RP. A mechanistic classification of closed, indirect fractures and dislocations of the lower cervical spine. Spine (Phila Pa 1976) 1982;7:1-27.

2. Eismont FJ, Arena MJ, Green BA. Extrusion of an intervertebral disc associated with traumatic subluxation or dislocation of cervical facets: case report. J Bone Joint Surg Am 1991;73:1555-60.

3. Allred CD, Sledge JB. Irreducible dislocations of the cervical spine with a prolapsed disc: preliminary results from a treatment technique. Spine (Phila $\mathrm{Pa}$ 1976) 2001;26:1927-30.

4. Levine AM. Facet fractures and dislocations. In: Levine AM, Eismont FJ, Garfin SR, Zigler JE, editors. Spine trauma. Philadelphia: WB Saunders; 1998. p.331-66.

5. Tribus CB. Cervical disc herniation in association with traumatic facet dislocation. Tech Orthop 1994;9:5-7.

6. Reindl R, Ouellet J, Harvey EJ, Berry G, Arlet V. Anterior reduction for cervical spine dislocation. Spine (Phila Pa 1976) 2006;31:648-52.

7. Nassr A, Lee JY, Dvorak MF, et al. Variations in surgical treatment of cervical facet dislocations. Spine (Phila Pa 1976) 2008;33:E188-93. 